\title{
Penile hyperexcitability with recurrent ejaculations as the presenting manifestation of a case of rabies
}

\author{
Z.F. Udwadia, F.E. Udwadia, P.P. Rao and F. Kapadia
}

The Rabies Ward, Sir J.J. Group of Teaching Hospitals, Bombay, India

\begin{abstract}
Summary: A patient with rabies presented with penile hypersensitivity and recurrent ejaculation. This preceded the more classical manifestations such as hydrophobia and aerophobia by approximately 8 hours. A review of the literature reveals that a variety of urogenital symptoms may rarely occur in rabies. Ours is however the first report documenting the occurrence of these symptoms at the onset of the disease in an autopsy proven case of rabies. Damage to the amygdaloid nucleus has been postulated to be the basis of these urogenital symptoms. This correlates well with our autopsy findings of extensive neuronal damage and Negri bodies in the temporal poles.
\end{abstract}

\section{Introduction}

We report the extremely rare occurrence of penile hyperexcitability and recurrent ejaculation as the initial clinical manifestation of a case of human rabies. This occurred about 8 hours before the more classical manifestations of rabies: hydrophobia, aerophobia and hyperexcitability were observed.

\section{Case report}

A 47 year old male was admitted with the chief complaints of recurrent ejaculation and hydrophobia. The patient, who was fully conscious and lucid at the time of admission and gave us his own history, confirmed that that morning he had hypersensitivity of the penis manifesting as recurrent erection and ejaculation occurring at the slightest touch. The hypersensitivity was so exquisite that the mere touch of his underwear against his penis was enough to trigger off spontaneous erection and ejaculation. There were no other urogenital symptoms. About 8 hours later he noticed hydrophobia for which he was admitted to a local hospital; he was then transferred to our rabies ward a few hours later when the diagnosis of rabies was entertained.

On questioning, there was a history of an unprovoked dog-bite on his left hand 3 months ago from a stray dog. Besides cleaning the wound and local dressings, the patient had not commenced

Correspondence: Z.F. Udwadia, M.D., 11-C, 'Il Palazzo', Ridge Road, Bombay 400 006, India

Accepted: 8 July 1987 prophylactic antirabies vaccine or serum therapy.

Examination revealed an anxious but fully conscious and alert patient. His vital parameters were all normal. Attempts to drink water provoked classical attacks of hydrophobia - later these attacks were provoked by the sight of water and about 6 hours thereafter aerophobia set in. The neurological examination was entirely normal with no abnormalities in muscle power, tone or reflexes.

Seven hours after admission he became violent, abusive and disoriented. Though the hydrophobia persisted the penile hyperexcitability had by this time spontaneously ceased.

An elective tracheostomy was done and the patient was sedated, curarised and put on a volume cycled respirator.

Important but anticipated complications that punctuated his 8 day hospital stay were hyperpyrexia (with rectal temperatures of $107^{\circ} \mathrm{F}$ ), paralytic ileus and the syndrome of inappropriate antidiuretic hormone secretion.

The patient died after sudden cardiac arrest. Post-mortem examination revealed extensive Negri body pathology in the brain. Swiss albino suckling mice were inoculated intracerebrally with suspension of the brain and spinal cord obtained at post-mortem and all showed typical signs of rabies.

\section{Discussion}

Our report documents the fairly typical clinical course and management of a patient with rabies.

(C) The Fellowship of Postgraduate Medicine, 1988 
The unique aspect of this case, however, is the unusual presentation with penile hyperexcitability and recurrent ejaculation. A variety of urogenital symptoms have been rarely reported in rabies. Penile pain has been reported as the earliest symptom of this disease. ${ }^{1,2}$ Priapism is another rare but well documented manifestation. ${ }^{3}$ Penile hypersensitivity as the earliest manifestation of the disease has only been reported in one previous case - though the diagnosis of rabies was not confirmed by serology or autopsy in that patient. ${ }^{4}$ In our patient as in the previously reported case the presenting manifestation of rabies was penile hypersensitivity with recurrent ejaculation. This preceded the occurrence of the more classical manifestations of rabies like hydrophobia, aerophobia and hyperexcitability by approximately 8 hours. The exact cause of the urogenital symptoms that may rarely accompany rabies is not clear. Recurrent ejaculation and even priapism has been postulated to be secondary to damage to the amygdaloid nucleus in the temporal pole. ${ }^{5}$ This correlates well

\section{References}

1. Bramley, J.E.D. Penile pain in rabies. Br Med J 1965, 2: 880.

2. Gosh, S. A case of rabies in England. Br Med J 1964, 2: $167-168$.

3. Babes, V. Traite de la Rage. J.V. Bailliere, Paris, 1912, pp 81-119.

4. Bhandari, M. \& Kumar, S. Penile hyperexcitability as the presenting symptom of rabies. Br J Urol 1986, 58: 224. with our autopsy findings of congestion of blood vessels and oedema in both frontal, temporal and anterior parietal lobes on gross examination. On microscopy, haematoxylin and eosin stained sections through the temporal poles and hippocampus revealed large numbers of Negri bodies in the Purkinje cells. Thus the clinico-pathological correlation was excellent.

In conclusion this report points out that recurrent ejaculation may be the earliest manifestation of rabies, and that in endemic areas a history of contact with dogs should be ascertained in any patient presenting with urogenital symptoms like penile pain, penile hyperexcitability and priapism of obscure aetiology.

\section{Acknowledgement}

The authors are grateful to Mr R. Khusrokhan for assistance in preparation of the manuscript.

5. Corey, L. Rabies and other rhabdoviruses. In: Braunwald, Isselbacher, Petersdorf, Wilson, Martin, Fanci (eds). Harrison's Principles of Internal Medicine, 11th edition. McGraw-Hill, USA, 1987, pp 712-717. 